\title{
Is replacement modality choice knowledge important in the non-renal multidisciplinary team? Experience from a single UK centre
}

\author{
Authors: Fatima Abdelaal, ${ }^{\mathrm{A}}$ Hatem $\mathrm{Ali}^{\mathrm{B}}$ and Jyoti Baharani ${ }^{\mathrm{C}}$
}

\begin{abstract}
Dialysis remains the mainstay treatment for patients with end stage renal disease. In the UK, there has been a significant decline in home dialysis despite its benefits and cost effectiveness. Patients with chronic kidney disease (CKD) are often known to other specialties who they may continue to consult when approaching dialysis. We wished to assess the knowledge of the non-renal multidisciplinary team (MDT) regarding home dialysis and establish whether further education was warranted. This was assessed using an online survey sent to specialties likely to deal with CKD patients. In total, 364 questionnaires were sent out with a $26.4 \%$ response rate. According to the survey responses, $\mathbf{8 1 . 5 \%}$ of non-renal MDTs lack confidence in discussing home dialysis options with patients and $74.55 \%$ feel that they need further education about home dialysis. Targeted education may increase home dialysis uptake by multimorbid CKD patients who have a consistent message delivered by all relevant healthcare teams about the benefits of home dialysis.
\end{abstract}

KEYWORDS: Chronic kidney disease, education, end stage renal disease, home dialysis, non-renal MDT

\section{Introduction}

Chronic kidney disease (CKD) is an increasing public health problem affecting $10-15 \%$ of the adult population. Kidney transplant is the optimum treatment in most patients with end stage renal disease (ESRD) as it improves quality of life and prolongs survival compared with patients on the transplant waiting list who remain on dialysis. ${ }^{1,2}$ However, renal transplantation may be contraindicated either for absolute or relative reasons. ${ }^{3-5}$

Dialysis remains the mainstay treatment for patients with ESRD who are not suitable for renal transplant or in whom transplant is contraindicated. The most widely used dialysis modality worldwide, including in the UK, is in-centre

\footnotetext{
Authors: ${ }^{A}$ specialty registrar in renal medicine, Birmingham

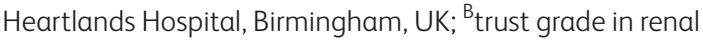
medicine, Birmingham Heartlands Hospital, Birmingham, UK; ${ }^{C}$ consultant physician and nephrologist, Birmingham Heartlands Hospital, Birmingham, UK
}

haemodialysis. ${ }^{1-3}$ Despite the possibility and the availability of home dialysis in the UK, in-centre haemodialysis remains the commonest modality. Home dialysis includes both peritoneal dialysis (PD) and haemodialysis. Home haemodialysis (HHD) can be carried out frequently as short daily dialysis or extended nocturnal dialysis. Similarly, PD can be applied overnight or 3-4 times a day depending upon the patients clinical requirements.

The number of patients on dialysis is increasing internationally by $7 \%$ per annum. ${ }^{6}$ The National Institute for Health and Care Excellence (NICE) in the UK emphasises the benefits of home-based dialysis and recommends it as the preferred option for suitable patients in their guidelines. ${ }^{7}$ Home dialysis is underutilised, but usage is variable worldwide - ranging from $9 \%$ penetration in the USA to $18 \%$ in the UK and up to $51.4 \%$ in New Zealand. ${ }^{8}$ Over the last 15 years, there has been a decline in home dialysis in health systems with previously high rates of home dialysis. ${ }^{9}$ One of the major factors contributing to the limited numbers on home dialysis is lack of awareness of this treatment option (either at clinician or patient level). ${ }^{10,11}$ Other reasons for the decline include economic and infrastructure barriers and the provider's impression that there are patient characteristics that contraindicate home dialysis. ${ }^{12}$ Home dialysis offers a variety of quality of life benefits, such as improved convenience, mental health wellbeing, rehabilitation, employment, reduced mortality and cost effectiveness.

The selection of dialysis modality is influenced by a number of factors - for example patient motivation, desire, education, medical history, physician and/or nurse bias and the ability to tolerate volume shifts. ${ }^{13,14}$

Among most patients, and especially if pre emptive transplantation is not possible, both PD and haemodialysis have a place in the patient's dialysis journey. ${ }^{14}$ Starting with PD has the advantages of increased commutative survival, continuous removal of uraemic toxins, preservation of residual renal functions, reduced risk of infection, greater patient satisfaction and lower cost. ${ }^{15,16}$ HHD should be the transition from PD. The final stop in this journey should be in-centre haemodialysis. It is evident, however, that currently this is not the route taken by the vast majority of dialysis patients.

Of all the renal replacement modalities, survival is generally highest in patients treated with HHD. ${ }^{17}$ The benefits of HHD are convenience, improvement of health and resource 
utilisation. However, HHD use is limited by lack of pre-dialysis education and training, lack of self-confidence, lack of family and social support, fear of self-cannulation and worries of catastrophic events. ${ }^{18}$ Nocturnal haemodialysis and short daily haemodialysis may be associated with improved patient survival compared with conventional in-centre haemodialysis. ${ }^{19}$ The FREEDOM (Following Rehabilitation, Economics and Everyday-Dialysis Outcome Measurements) study showed marked improvement in both physical and mental healthrelated quality of life measures among patients receiving home short daily dialysis assessed by the 36-Item Short Form health survey. ${ }^{20}$ A survey in 2003-04 among 150 HHD patients at a Sydney dialysis centre performing long hours nocturnal haemodialysis showed that patients who dialysed at night and for longer hours experienced an improved sense of wellbeing, more dietary freedom, increased energy levels, an increased opportunity for employment, improved blood parameters and, overall, an improved lifestyle. ${ }^{21}$

In current practice, in-centre haemodialysis is commonly the first and only link in the chain for most ESRD patients and this is undoubtedly in part responsible for the static outcome of patients on dialysis over the last few decades.

Many patients commencing dialysis have four or more comorbidities, most of which are present for a number of years prior to reaching the advanced stages of CKD. Patients may not be referred to renal services until they approach CKD4/5 and may receive some information regarding renal replacement therapy (RRT) options from their primary medical team. Some of this information may well be biased and not based on current evidence-based practice. An example to illustrate this point is a diabetic patient who will be fairly close to his diabetic nurse/ doctor and may ask them for advice when faced with the dilemma of dialysis modality choice. It is important that the seed for treatment at home is sown early in the CKD patient's journey. Sharing adequate information on treatment options and allowing sufficient time to discuss management alternatives with families and carers is of great importance among CKD patients. Therefore, knowledge about home therapy in these teams is of importance and we have a vested interest in educating these teams.

It is important to highlight that, in some CKD patients, dialysis treatment might not be suitable or in the patient's best interest and a conservative management approach might be the best way forward.

There are no data about home dialysis awareness among the nonrenal multidisciplinary team (MDT) despite the high prevalence of CKD among a number of medical specialties. The aim of this survey is to assess home dialysis awareness among non-renal MDTs in medical specialties that are likely to care for renal patients in view of their comorbidities or their underlying cause of CKD and to establish whether further education is required.

\section{Materials and methods}

\section{Study design}

Home dialysis awareness among non-renal healthcare workers was assessed using an online web-based questionnaire (SurveyMonkey), which was active for 30 days (28 January to 27 February 2016). The survey was sent by email to 364 non-renal healthcare workers in different medical specialties at Birmingham Heartlands Hospital. The list of people who were deemed appropriate for the survey was identified from the hospital intranet and through medical secretaries. The medical specialties chosen included geriatrics, diabetes and endocrinology, haematology, urology, cardiology and vascular surgery. These specialties were selected as they were likely to see renal patients in view of their associated comorbidities or the underlying cause of the patients' CKD.

Emails of medical staff in the relevant departments targeted in this study were collected from the hospital intranet. Emails have been sent to staff with the survey link. The email included a brief discussion about the background and the objectives of the study. By responding to the survey, correspondence were considered to have consented to participate in this study. This study required no ethical approval.

\section{Questionnaire}

The questionnaire focused on assessing home dialysis knowledge and views of patient outcomes among the non-renal MDT. It also aimed at identifying whether further education is required and the preferred methods for delivering that education.

\section{Statistics}

Results were analysed using descriptive statistics (frequencies and percentages) through the facilities on the web-based survey.

\section{Results}

The survey was sent to 364 non-renal healthcare workers at Birmingham Heartlands Hospital in specialties who are likely to care for renal patients as described above; 19 people opted out and 4 emails bounced back. In total, 90 people responded to the survey ( 86 complete and 4 partial responses). $47.19 \%$ of respondents were doctors and $52.81 \%$ were healthcare workers (nurses $16.9 \%$, healthcare assistants 2.2\%, dieticians $7.9 \%$, pharmacists $3.4 \%$ and others $22.5 \%$ ); $69.32 \%$ of respondents were working in common specialties dealing with CKD patients (geriatrics $15.9 \%$, cardiology $14.8 \%$, haematology $10.2 \%$, endocrinology $10.2 \%$, urology $10.2 \%$ and vascular surgery $8 \%$ ). In total, 60 respondents $(67.42 \%)$ said they come across patients with CKD approaching the need for RRT or ESRD that are on home dialysis - either PD or HHD; of these, only $18.46 \%$ provide advice on dialysis modality (Fig 1). Approximately half of respondents (49.23\%) felt that they have the least influence on their patients' RRT modality choice (Fig 2). Only $31.66 \%$ of the respondents worry about the poor compliance among patients on home therapy (Fig 3A). The majority of respondents (77.58\%) encourage their patients to consider home dialysis because of its flexibility (Fig 3B). 38.6\% of non-renal healthcare workers consider that the high quality of professional care that in-centre dialysis patients receive is more important than the flexibility that they get with home dialysis (Fig 3C). Half of respondents (50\%) consider PD as the easiest modality for patients when travelling, while $44.44 \%$ consider HHD so (Figure 3D). Most respondents $(92.98 \%)$ agree that with proper training, most patients can learn to do PD or HHD safely and effectively (Fig $3 \mathrm{E}$ ).

Pre-dialysis nurses are considered to have the most impact on patient's choice of dialysis modality by respondents (40.39\%). This is followed by $31.58 \%$ and $24.56 \%$ of respondents who think that nephrologists and the patients themselves are the ones who have the most impact on modality choice. When 

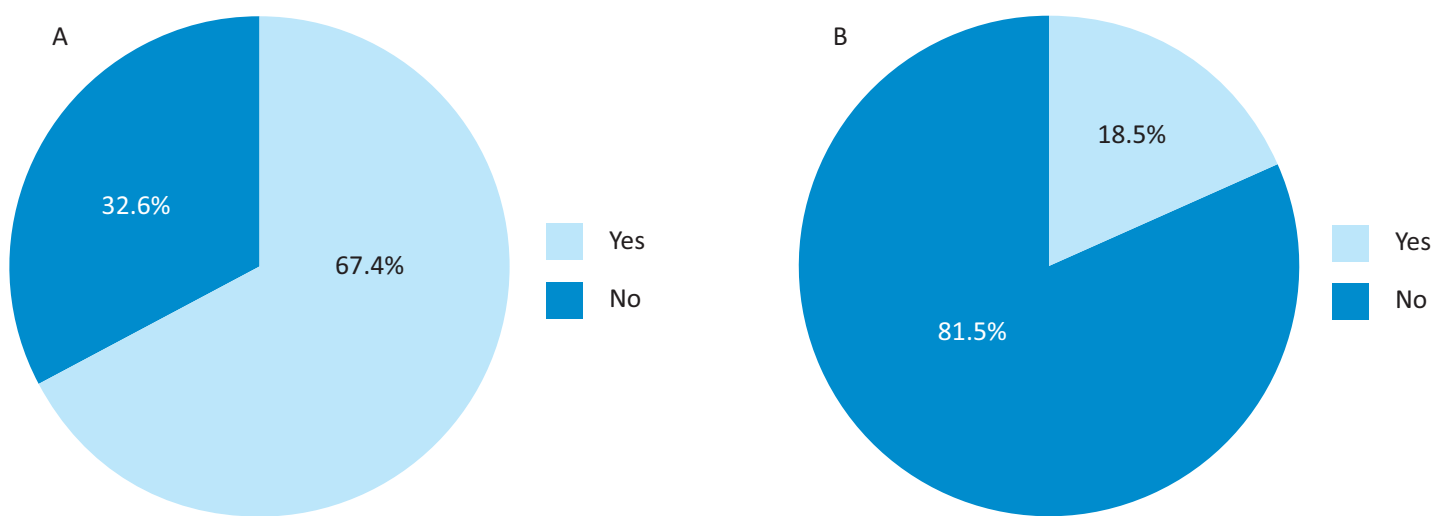

Fig 1. Percentage of patients with chronic kidney disease seen by other specialties and advice given regarding renal replacement therapy modality. A-in your specialty, do you come across patients with chronic kidney disease approaching the need for renal replacement therapy? B - do you advise patients on dialysis modality?

asked which modality they would choose if they required dialysis themselves, $58.93 \%$ and $23.21 \%$ of respondents would opt for HHD or PD, respectively. Half of respondents think that home dialysis has a better outcome; of these, $53.85 \%$ thought so because of a lower rate of hospitalisation among home dialysis patients (Fig 3F). A majority of respondents $(70 \%)$ felt that their knowledge about HHD was poor and $74.55 \%$ felt that they would benefit from further education about home dialysis (Fig 4A). They also felt that online education $(65.85 \%)$ followed by lectures $(41.46 \%)$, videos $(31.71 \%)$ and leaflets $(24.39 \%)$ would be the best methods to provide further information about home dialysis (Fig 4B).

\section{Discussion}

To our knowledge, there are no studies on home dialysis awareness among the non-renal MDT. Moreover, despite the high prevalence of CKD, there are no data on its prevalence among the medical specialties chosen for this survey. It seems that there is a high prevalence of CKD among medical specialties and this could be found in a cross-sectional study among patients attending a specialist diabetes clinic in Jamaica between 2009 and 2010 that showed that the overall prevalence of CKD

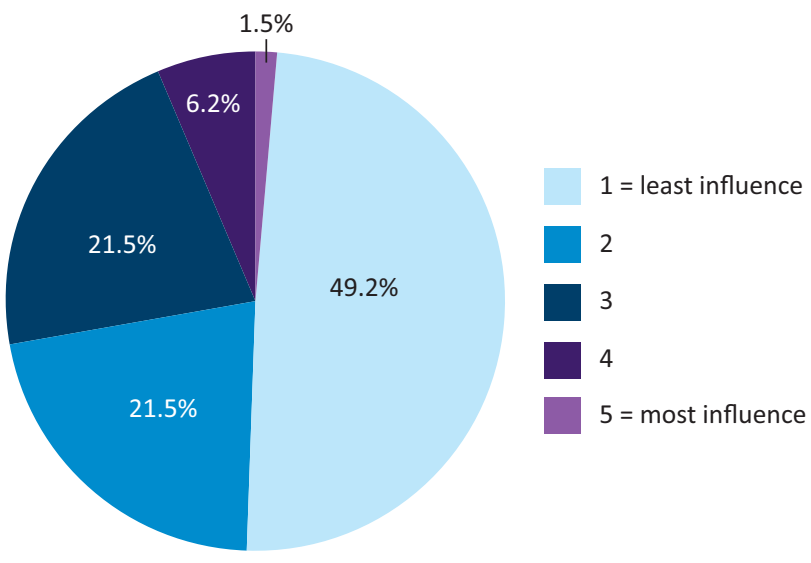

Fig 2. Influence of the non-renal multidisciplinary team on patients' renal replacement modality of choice. Survey respondents were asked to rate how much influence they think they have on a scale on 1-5 where 1 is the least influence and 5 is the most influence. was $86.3 \%$. Based on KDIGO (Kidney Disease Improving Global Outcomes) risk categories, $50.8 \%$ and $17.4 \%$ were at high risk or very high risk of adverse outcomes, respectively. ${ }^{22}$ Our study has shown that $69.32 \%$ of the chosen specialties (geriatrics, diabetes and endocrinology, haematology, urology, cardiology and vascular surgery) deal with CKD patients.

Patients with CKD are faced with complex decision making throughout their treatment journey, especially RRT options, which may impact on how they live from day to day. ${ }^{23}$ When Patients reach the need for RRT, they may continue to consult their primary medical team whom they have known for a number of years. Equally, they might be preoccupied by the primary discussion about RRT options, which is less likely to cover home therapy, resulting in patients opting for in-centre haemodialysis.

Patients make their decisions based on the information provided by healthcare workers. We speculate that CKD patient are likely to opt for home dialysis if they get consistent information regarding its benefits from all the clinicians involved in their care. Moreover, peer influence, timing of information and simultaneous creation of vascular access may affect patient decision making and explain why patients often continue on the initiated treatment modality. ${ }^{24}$

A limited number of the non-renal MDT $(18.46 \%)$ give RRT counselling to their patients and almost half of the respondents felt that they have the least influence on the patient's selection of RRT modality. We have looked at some themes to assess knowledge about home dialysis, including compliance, flexibility, convenience, travel and outcome. Our study has highlighted that home dialysis awareness among the nonrenal MDT is poor and that $74.55 \%$ felt that they need further education about home dialysis. Surprisingly, the majority of the non-renal MDTs in our study would opt for home therapy (HHD 58.93\% and PD 23.21\%) if they required dialysis themselves despite their limited knowledge about it.

Providing home dialysis needs nephrologists with experience in the technique as well as nursing staff capable of providing the training; this should be underpinned by a well-functioning pre-dialysis programme. ${ }^{25}$ The majority of the non-renal MDT (92.98\%) agree that, with proper training, most patients can learn to perform PD or HHD safely and effectively. Our study showed that $40.93 \%$ of the non-renal MDT thinks that pre-dialysis nurses have the most influence on patient modality choice. 

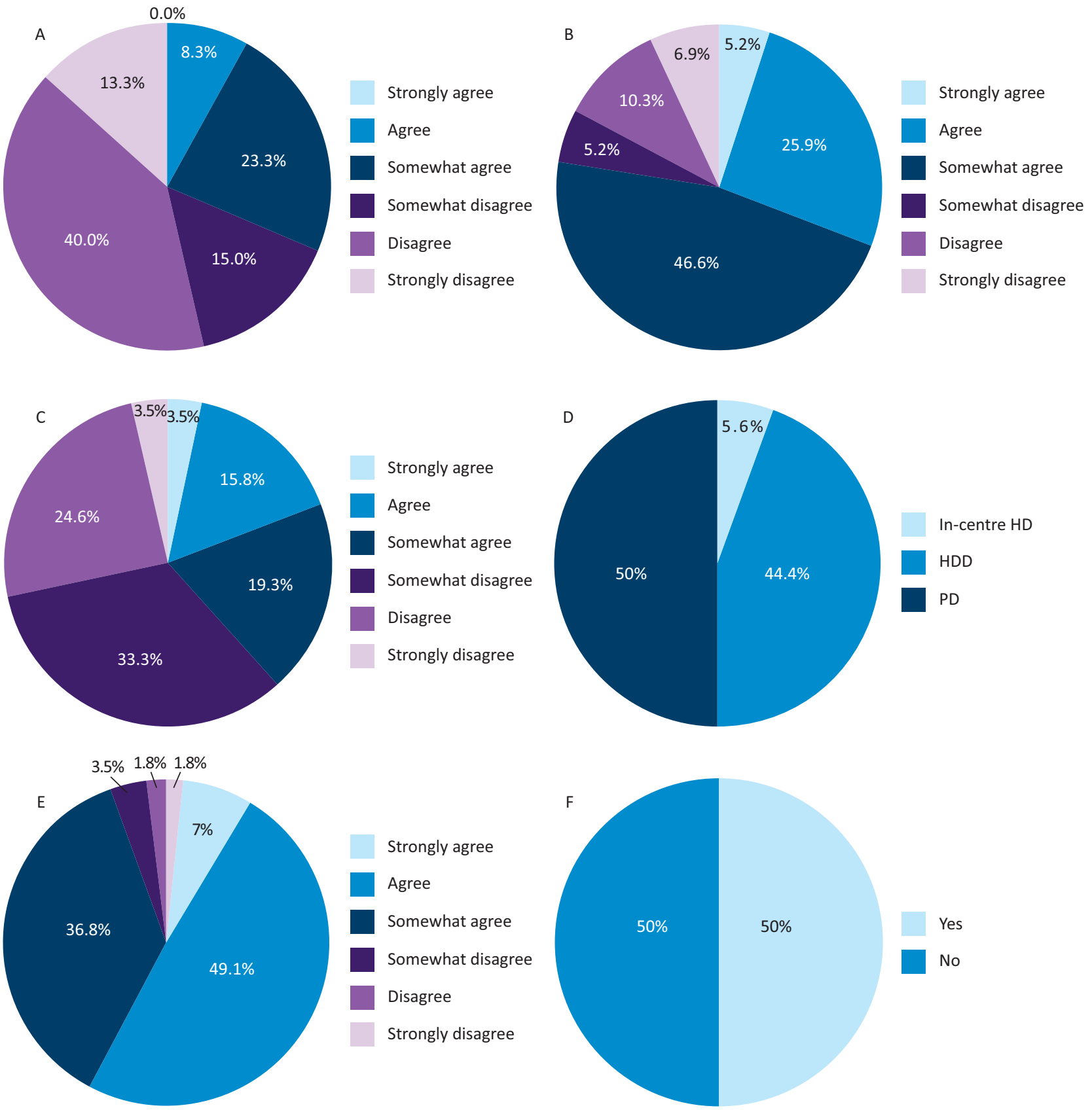

Fig 3. Themes used to assess respondents' knowledge of home dialysis. A - I worry that home dialysis patients (PD or HHD) will not comply with their treatment programme. B - I encourage patients to consider home dialysis (PD or HDD) because it gives them the flexibility to dialyse at any time that is convenient to them. $\mathrm{C}$ - The high quality of professional care that in-centre dialysis patients receive is more important than the flexibility that they get with home dialysis (PD or HDD). D - which dialysis modality do you think makes travelling easiest? $\mathrm{E}$ - with proper training, most patients can learn to do PD or HDD safely and effectively. $\mathrm{F}$ - do you think that home dialysis (PD or HDD) has a better outcome than in-centre dialysis? For $\mathrm{A}-\mathrm{C}$ and $\mathrm{E}$, respondents were asked to rate how much they agree with these statements. $\mathrm{HDD}=$ home haemodialysis; $\mathrm{PD}=$ peritoneal dialysis

In 2012, Tong et al ${ }^{26}$ conducted a semi-structured interview among nephrologists and in-centre dialysis nurses to explore their beliefs and attitudes about HHD, and identify barriers to HHD and strategies to increase its uptake. The study concluded that programmes that provide clinicians with direct experience of HHD could increase acceptance and motivation for home-based haemodialysis. ${ }^{26}$ We think that wider awareness about home dialysis therapy among the non-renal MDT - who see a significant number of CKD patients - might increase the uptake of home dialysis, especially in those patients that might be under another medical team for a good number of years who have therefore gained their confidence and trust. Hence, nephrologists' efforts should be focused on educating themselves and their colleagues about home therapies. This is expected to classify CKD patients at an early stage to identify suitable candidates for home therapy and 

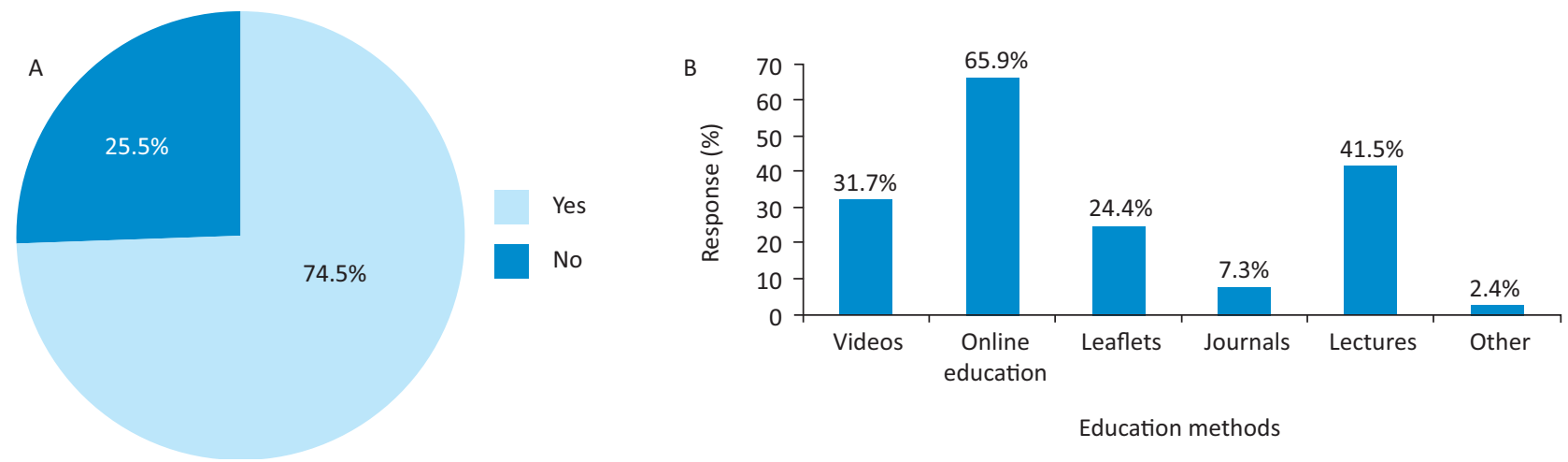

Fig 4. Dialysis education. A - do you think that you would benefit from more education about home dialysis? B - what would be the best way(s) to provide you with further information about different dialysis modalities?

introduce patients to different dialysis modalities. ${ }^{27}$ In 2012, a study done at Birmingham Heartlands Hospital highlighted important factors influencing the ESRD patients' choice of treatment modality and drew attention to the importance of good information provision and pre-dialysis education in empowering socially able patients to choose self-care therapies. $^{28}$

We speculate that lack of awareness and limited knowledge about home dialysis among the non-renal MDT may reduce the uptake of home dialysis. A 2015 study concluded that continuing nursing education initiatives can modify the opinions of in-centre haemodialysis nurses towards home modalities and should be one of the strategies aimed at promoting home dialysis. ${ }^{14}$ In our study, the non-renal MDT has identified different education methods but the most popular were online education $(65.85 \%)$, lectures $(41.46 \%)$, videos $(31.71 \%)$ and leaflets (24.39\%). A 2016 systematic review of educational interventions among CKD patients highlighted that a well-designed, interactive, frequent and multifaceted educational intervention that includes both individual and group participation may improve knowledge, self-management and patient outcomes. ${ }^{29}$ Half of patients who receive pre-dialysis education choose a home dialysis modality. Close correlation between nephrologists, patients and healthcare providers is required to increase the uptake of home dialysis. ${ }^{30}$

\section{Limitations}

Despite multiple reminders, the response rate to our survey was only $26.1 \%$. This may reflect a lack of awareness, that people felt their knowledge was too limited to participate in the survey or that they may have perceptions that are not in favour of home dialysis.

To our knowledge, this is the first study looking at home dialysis awareness among the non-renal MDT. The limitations of our study include the small sample size, the single-centre nature and the restriction to certain specialties. Furthermore, the analysis used in this study is purely descriptive and therefore might have resulted in some biased results. We think that further studies can be conducted to include GPs and a comparison should be done between awareness among renal and non-renal MDTs.

\section{Conclusions}

This survey demonstrates that a significant number of nonrenal healthcare workers do come across patients with CKD in their specialties. However, knowledge of home dialysis among non-renal healthcare professionals is poor and they lack the confidence to discuss treatment modalities with CKD patients. There is a clear need for further education about home dialysis, which can be facilitated by the nephrology department in different formats. This is likely to increase the number of multimorbid CKD patients opting for home therapy and therefore may improve the patient's outcome and quality of life.

\section{Conflicts of interest}

The authors declare no conflicts of interest.

\section{Author contributions}

FA contributed to designing the questionnaire used in this study, and collection, analysis and interpretation of data. She also performed the literature review around the topic and wrote the submitted article. HA made a substantial contribution to designing the questionnaire and to collection of the data. HA also contributed to the literature review and writing the manuscript. JB critically revised the work at all stages, from the questionnaire used in this survey up to the final article submission.

\section{References}

1 Huang Y, Samaniego M. Pre-emptive kidney transplantation: has it come of age? Nephrol Ther 2012;8:428-32.

2 Suthanthiran M, Strom TB. Renal transplantation. N Engl J Med 1994;331:365-76.

3 McKay DB, Milford EL, Sayegh MH. Clinical aspects of renal transplantation. In: Brenner BM, Rector FC (eds). The Kidney, 5th edn. Philadelphia: Saunders, 1995.

4 Knoll G, Cockfield S, Blydt-Hansen T et al. Canadian Society of Transplantation: consensus guidelines on eligibility for kidney transplantation. CMAJ 2005;173:S1-25.

5 Kasiske BL, Cangro CB, Hariharan S et al. The evaluation of renal transplantation candidates: clinical practice guidelines. $\mathrm{Am} J$ Transplant 2001; 1(Suppl 2):3-95.

6 Grassmann A, Gioberge S, Moeller S et al. ESRD patients in 2004: global overview of patient numbers, treatment modalities and associated trends. Nephrol Dial Transplant 2005;20:2587-93. 
7 National Institute for Health and Care Excellence. Guidance on home compared with hospital haemodialysis for patients with endstage renal failure. NICE technology appraisal No 48. London: NICE, 2002.

8 Collins AJ, Foley RN, Chavers B et al. United States Renal Data System 2013 annual data report: Atlas of chronic kidney disease and end-stage renal disease in the United States. Am J Kidney Dis 2014;63(Suppl 1):e1-e478.

9 MacGregor MS, Agar JW, Blagg CR. Home haemodialysis-international trends and variation. Nephrol Dial Transplant 2006;21:1934-45.

10 Bennett PN, Jaeschke S, Sinclair PM et al. Increasing home dialysis knowledge through a web-based e-learning program. Nephrology 2014;6:345-51.

11 Hope J. A patient perspective on the barriers to home dialysis. J Ren Care 2013;39(Suppl 1):3-8.

12 Qamar M, Bender F, Rault R, Piraino B. The United States' perspectives on home dialysis. Adv Chronic Kidney Dis 2009;16:189-97.

13 Miskulin DC, Meyer KB, Athienites NV et al. Comorbidity and other factors associated with modality selection in incident dialysis patients: the CHOICE Study. Choices for Healthy Outcomes in Caring for End-Stage Renal Disease. Am J Kidney Dis 2002;39:324-36.

14 Phillips M, Wile C, Bartol C et al. An education initiative modifies opinions of haemodialysis nurses towards home dialysis. Can J Kidney Health Dis 2015;2:16.

15 Biesen WV, Davies S, Lameire N. An integrated approach to endstage renal disease. Nephrol Dial Transplant 2001;16:7-9.

16 Termorshuizen F, Korevaar JC, Dekker FW et al. Hemodialysis and peritoneal dialysis: comparison of adjusted mortality rates according to the duration of dialysis: analysis of The Netherlands Cooperative Study on the Adequacy of Dialysis 2. J Am Soc Nephrol 2003;14:2851-60.

17 Masterson R. The advantages and disadvantages of home hemodialysis. Hemodial Int 2008;12 (Suppl 1):S16-20.

18 McLaughlin K, Manns B, Mortis G et al. Why patients with ESRD do not select self-care dialysis as a treatment option. Am J Kidney Dis 2003;41:380-5.

19 Pauly RP. Nocturnal home hemodialysis and short daily hemodialysis compared with kidney transplantation: emerging data in a new era. Adv Chronic Kidney Dis 2009;16:169-72.
20 Finkelstein FO, Schiller B, Daoui R et al. At-home short daily hemodialysis improves the long-term health-related quality of life. Kidney Int 2012;82:561-9.

21 Bolgg A, Hyde C. Enhancing lifestyle through home haemodialysis. J Ren Care 2006;32:179-82.

22 Ferguson TS, Tulloch-Reid MK, Younger-Coleman NO et al. Prevalence of chronic kidney disease among patients attending a specialist diabetes clinic in Jamaica. West Indian Med J 2015;64:201-8.

23 Elias MF, Dore GA, Davey A. Kidney disease and cognitive function. Contrib Nephrol 2013;179:42-57.

24 Morton RL, Tong A, Howard $\mathrm{K}$ et al. The views of patients and carers in treatment decision making for chronic kidney disease: systematic review and thematic synthesis of qualitative studies. BMJ 2010;340:c112.

25 Hoenich NA, Ronco C. Perspectives in home hemodialysis therapy. Contrib Nephrol 2011;171:25-9.

26 Tong A, Palmer S, Manns B et al. Clinician beliefs and attitudes about home haemodialysis: a multinational interview study. BMJ Open 2012;2:e002146.

27 Mehrotra R, Marsh D, Vonesh E, Peters V, Nissenson A. Patient education and access of ESRD patients to renal replacement therapies beyond in-center hemodialysis. Kidney Int 2005;68:378-90.

28 Chanouzas D, Ng K, Fallouh B and Baharani J. What influences patient choice of treatment modality at the pre-dialysis stage? Nephrol Dial Transplant 2012;27:1542-7.

29 Lopez-Vargas PA, Tong A, Howell M, Craig JC. Educational interventions for patients with CKD: a systematic review. Am J Kidney Dis 2016;68:353-70.

30 Schiller B, Munroe H, Neitzer A. Thinking outside the box - identifying patients for home dialysis. NDT Plus 2011;4(Suppl 3):iiil1-iiil3.

Address for correspondence: Dr Fatima Abdelaal, Department of Renal Medicine, Heartlands Hospital, Bordesley Green East, Birmingham B9 5SS, UK, Email: Fatima.Abdelaal@heartofengland.nhs.uk 